\title{
Adaptive Backstepping for Uncertain Systems With Time-Delay On-Line Update Laws
}

\author{
Delphine Bresch-Pietri, Jonathan Chauvin, Nicolas Petit
}

\begin{abstract}
This paper addresses the general problem of the equilibrium regulation of potentially unstable linear systems with an unknown input time-delay and unknown parameters in the plant. We extend recent results from the literature where such systems are treated using a backstepping approach applied to a distributed parameters system representation of the delay. Furthering previous approaches, our contribution concerns on-line adaptation of the delay. We develop a local asymptotic convergence result for single-input systems. It is illustrated in simulations on the control of the Air-Fuel Ratio in Spark Ignition engines. The results on this particular example stress the merits of the proposed control algorithm which, with reduced implementation difficulties, reveals sympathetic to online applications.
\end{abstract}

\section{INTRODUCTION}

Time-delay phenomena, commonly introduced by actuators and sensors involved in feedback loops, and often modeled as lags at the system input, can be encountered in many engineering field of interest and represent a challenge in control design (see e.g. [13]).

Following the well-known Smith Predictor [14], such problems are usually treated with predictor-like feedback approaches, based on the Arstein reduction (see [1]) and designed to overcome some of the inherent problems of the conventional Smith Predictor [12]. Among these problems are its lack of robustness to delay or plant parameters mismatches.

Recently, (see [7],[8],[9]), a new approach of this type has been proposed to treat such systems. It is a form of backstepping boundary control for partial differential equations (PDEs), modeling the actuator delay as a transport process. The main merit of this approach is to enable the useage of systematic tools to design a general adaptive control scheme accounting for uncertainties including unknown delay [4] [5]. In particular, these tools can be used for systems with an uncertain delay of significant length, for which it is well known that the Padé approximation, which also yields a formulation compliant with adaptive control techniques, is not adapted (see e.g. [2]).

In this paper, we apply these tools to control a class of potentially open-loop unstable systems, with an uncertain (single) input time-delay and uncertain parameters in the

D. Bresch-Pietri (corresponding author) is a $\mathrm{PhD}$ Candidate in Mathematics and Control at MINES ParisTech, 60, Bd St-Michel, 75272 Paris, Cedex 06, France. Email : delphine.bresch-pietri@mines-paristech.fr

J. Chauvin is with the Département Contrôle, Signal et Système in IFP Energies Nouvelles, 1-4 Av. du Bois Préau, 92852 Rueil Malmaison, France

N. Petit is with the Centre Automatique et Systèmes, Unité Mathématiques et Systèmes at MINES ParisTech, 60 Bd St Michel, 75272 Paris, France plant. Following [3], we extend the design presented in [5] by alleviating the assumption that the full actuator state (i.e. the past values of the input) is known over an interval equal to the delay, like usually considered in [5] [8] [9]. This lack of information does not prevent us from stabilizing the system around the tracked trajectory, but this is done at the expense of global asymptotic stability, which becomes only local (i.e. we require that the estimates of the delay and the plant parameter are sufficiently close to their true values and that both the system state and the actuator state are close to the set point).

This modification in the adaptive control makes it sympathetic with most engineering problems, where the actuator state is not always measurable, or at least often highly uncertain. Furthering the approach proposed in [3], we account for delay adaptation laws, of practical importance for applications. As is proven, the arising strategy is compatible with various such update laws. This is the main contribution of this paper. An illustrative example is provided.

Due to the introduction of delay adaptation laws, significant changes must be considered in the proof technique, compared to [3]. These are discussed.

The paper is organized as follows. In Section II, we describe the general framework of the problem under consideration and briefly discuss it before presenting in Section III the general adaptive control strategy we propose. Two classes of delay update laws are considered. In Section IV, we prove the convergence properties of the control and illustrate it in Section V, with simulations of the control of the Air/Fuel Ratio (AFR) in Spark-Ignition (SI) engines.

\section{Problem Statement and Assumptions}

Consider the following potentially open-loop unstable delay system

$$
\begin{aligned}
& \dot{X}(t)=A(\theta) X(t)+B(\theta) U(t-D) \\
& Y(t)=C X(t),
\end{aligned}
$$

where $X \in \mathbb{R}^{n}$ is the state and $U$ is a scalar input. $D>0$ is an unknown (potentially long) constant delay and we assume that the system matrix $A(\theta)$ and the input vector $B(\theta)$ are linearly parametrized under the form

$$
A(\theta)=A_{0}+\sum_{i=1}^{p} A_{i} \theta_{i} \text { and } B(\theta)=B_{0}+\sum_{i=1}^{p} B_{i} \theta_{i},
$$

where $\theta$ is an unknown constant vector of parameters belonging to a convex closed set $\Pi=\{\theta \in \Pi \mid \mathscr{P}(\theta) \leq 0\} \subset$ $\mathbb{R}^{p}$, where $\mathscr{P}: \mathbb{R}^{p} \rightarrow \mathbb{R}$ is a smooth convex function. 
Following [5], several assumptions are made.

Assumption 1: The convex closed set $\Pi$ is known and bounded. An upper bound $\bar{D}$ and a lower bound $\underline{D}>0$ of the delay $\mathrm{D}$ are known.

Assumption 2: For a given set point $Y^{r}$, we assume that there exists known functions $X^{r}(\theta)$ and $U^{r}(\theta)$ continuously differentiable in the parameter $\theta \in \Pi$ which satisfy, for all $\theta \in \Pi$,

$$
0=A(\theta) X^{r}(\theta)+B(\theta) U^{r}(\theta), \quad Y^{r}=C X^{r}(\theta)
$$

Assumption 3: We assume that the pair $(A(\theta), B(\theta))$ is controllable for each $\theta \in \Pi$ and that there exists a triple of vector/matrix functions $(K(\theta), P(\theta), Q(\theta))$ such that

i) $P(\theta)$ and $Q(\theta)$ are positive definite and symmetric for each value of $\theta \in \Pi$

ii) the following Lyapunov equation is satisfied for $\theta \in \Pi$

$$
P(\theta)(A+B K)(\theta)+(A+B K)(\theta)^{T} P(\theta)=-Q(\theta)
$$

iii) $(K, P) \in C^{1}(\Pi)^{2}$ and $Q \in C^{0}(\Pi)$.

Assumption 4: The following quantities are well-defined

$$
\begin{aligned}
& \underline{\lambda}=\inf _{\theta \in \Pi} \min \left\{\lambda_{\min }(P(\theta)), \lambda_{\min }(Q(\theta))\right\} \\
& \bar{\lambda}=\sup _{\theta \in \Pi} \lambda_{\max }(P(\theta))
\end{aligned}
$$

The control objective is to have system (1) track the set-point $Y^{r}$ through a full-state feedback. Among these assumptions, only one is truly restrictive: Assumption 3 requires the equivalent delay-free form of the system (1) to be controllable. This is a reasonable assumption to guarantee the possibility of regulation about the constant reference $Y^{r}$. Assumptions 1 and 2 are formulated for well-posedness of the problem. Finally, Assumption 4 is formulated for Lyapunov design purposes only. We illustrate the feasibility of these assumptions in the application treated below.

As a final remark, we wish to stress that neither the considered reference $U^{r}$, nor the state reference $X^{r}$ depends on time or delay, because the reference $Y^{r}$ is constant. This point is crucial in the control design.

\section{Control Design}

We now present the control method we propose. It is a generalization of the approach [3], in the sense that multidimensional state are considered and, most importantly, delay adaptation laws (see Assumption 5 below) are incorporated into the convergence analysis.

We start our analysis by introducing the distributed input $u(x, t)=U(t+D(x-1)), x \in[0,1]$. The plant (1) can be represented under the form

$$
\begin{aligned}
\dot{X}(t) & =A(\theta) X(t)+B(\theta) u(0, t) \\
D u_{t}(x, t) & =u_{x}(x, t) \\
u(1, t) & =U(t)
\end{aligned}
$$

where the delay is accounted for by the transport equation whose speed of propagation is $1 / D$. Unfortunately, because this speed is uncertain, even if the applied input $U(t)$ is fully known, one cannot deduce the value of $u(x, t)$ for each $x \in[0,1]$ from it. Therefore, we introduce an estimate

$$
\hat{u}(x, t)=U(t+\hat{D}(t)(x-1))
$$

of the distributed input, using an estimate $\hat{D}$ of the delay. Consider the following error variables

$$
\begin{aligned}
\tilde{X}(t) & =X(t)-X^{r}(\hat{\theta}) \\
\tilde{U}(t) & =U(t)-U^{r}(\hat{\theta}) \\
e(x, t) & =u(x, t)-u^{r}(\hat{\theta}) \\
\hat{e}(x, t) & =\hat{u}(x, t)-u^{r}(\hat{\theta}) \\
\tilde{e}(x, t) & =e(x, t)-\hat{e}(x, t)=u(x, t)-\hat{u}(x, t)
\end{aligned}
$$

where $\hat{\theta}$ is an estimate of the parameter $\theta$. In details, (11)(14) all account for the estimation error of the unknown parameter, with (11) representing the state tracking error and (12)-(14) the input tracking error, while (15) is the estimation error of the distributed input.

The infinite-dimensional state of the system and the actuator estimation error are fully described by the vector $(\tilde{X}, e, \hat{e})$; other choices can be done, but this one is particularly suitable in the Lyapunov analysis of convergence.

When both the delay and the parameters of the system are known, the following controller (see [1]) achieves asymptotic stabilization of system (1) toward 0

$$
U(t)=K X_{P}(t+D)=K\left(e^{A D} X(t)+\int_{t-D}^{t} e^{A(t-s)} B U(s) d s\right)
$$

This controller can be viewed as a delay-version of the delay-free controller $U(t)=K X(t)$, where $X_{P}(t+D)$ should be understood as a D-units of time ahead prediction of the system state, starting from $X(t)$ as initial condition, and driven by the control history over the $D$-units of time window. This control has been interpreted in [7] as the result of a backstepping transformation of the transport partial differential equation (PDE) (9) ${ }^{1}$. We follow this analysis, and the one pursued in [4] and [5]. We employ here the control law

$$
\begin{aligned}
U(t)= & U^{r}(\hat{\theta})+K(\hat{\theta}) \tilde{X}_{P}(t+\hat{D}) \\
= & U^{r}(\hat{\theta})-K(\hat{\theta}) X^{r}(\hat{\theta})+K(\hat{\theta})\left[e^{A(\hat{\theta}) \hat{D}} X(t)\right. \\
& \left.+\hat{D}(t) \int_{0}^{1} e^{A(\hat{\theta}) \hat{D}(1-y)} B(\hat{\theta}) \hat{u}(y, t) d y\right],
\end{aligned}
$$

based on the certainty equivalence principle. The update law $\hat{\theta}$ is chosen as

$$
\begin{aligned}
\dot{\hat{\theta}}(t) & =\gamma_{\theta} \operatorname{Proj}_{\Pi}\left(\tau_{\theta}(t)\right) \\
\tau_{\theta, i}(t) & =h(t) \times\left(A_{i} X(t)+B_{i} u^{r}(\hat{\theta})\right) \\
h(t) & =\left(\frac{\tilde{X}(t)^{T} P(\hat{\theta})}{b_{2}}-\hat{D} K(\hat{\theta}) \int_{0}^{1}(1+x)[\hat{w}(x, t)\right. \\
& \left.\left.+A(\hat{\theta}) \hat{D} \hat{w}_{x}(x, t)\right] e^{A(\hat{\theta}) \hat{D} x} d x\right)
\end{aligned}
$$

\footnotetext{
${ }^{1}$ this transformation is made to convert the plant (8)-(10) into the target system $\dot{X}(t)=(A+B K)(\theta) X(t)+B(\theta) w(0, t)$

$D w_{t}(x, t)=w_{x}(x, t)$ with the boundary condition $w(1, t)=0$.
} 
with $\gamma_{\theta}>0,1 \leq i \leq p$ and where the transformed estimate state of the actuator satisfies the following Volterra integral equation of the second kind

$$
\begin{aligned}
\hat{w}(x, t)= & \hat{e}(x, t)-\hat{D} \int_{0}^{x} K(\hat{\theta}) e^{A(\hat{\theta}) \hat{D}(x-y)} B(\hat{\theta}) \hat{e}(y, t) d y \\
& -K(\hat{\theta}) e^{A(\hat{\theta}) \hat{D} x} \tilde{X}(t)
\end{aligned}
$$

In (19), the matrix $P$ is the one considered in Assumption 3 , the constant $b_{2}$ is chosen such that $b_{2} \geq$ $8 \sup _{\theta \in \Pi}|P B(\theta)|^{2} / \underline{\lambda}$, and $\operatorname{Proj}_{\Pi}$ is the standard projector operator onto the convex set $\Pi$

$\operatorname{Proj}_{\Pi}\left\{\tau_{\theta}\right\}=\tau_{\theta}\left\{\begin{array}{l}I, \quad \hat{\theta} \in \prod^{\circ} \text { or } \nabla_{\hat{\theta}} \mathscr{P}^{T} \tau_{\theta} \leq 0 \\ I-\frac{\nabla_{\hat{\theta}} \mathscr{P} \nabla_{\hat{\theta}} \mathscr{P} T}{\nabla_{\hat{\theta}} \mathscr{P}^{T} \nabla_{\hat{\theta}} \mathscr{P}}, \hat{\theta} \in \partial \Pi \text { and } \nabla_{\hat{\theta}} \mathscr{P}^{T} \tau_{\theta}>0\end{array}\right.$

From a comparison of (17)-(22) to the corresponding equation in [5], one can notice that the main difference consists in the use of the estimate actuator state $\hat{u}$ and actuator tracking error $\hat{e}$ (instead of the unmeasured value $u$ and $e$ ) respectively in the control law and the transformed state of the actuator. Two consequences arise from this design. First, the expression of the parameter update law (18)-(20) involves a spatial derivative of the transformed actuator state, which implies the uses of a $H_{1}$-norm of the transformed actuator state in the Lyapunov analysis, as it will appear in the following section. Secondly, the result stated in Theorem1 involves the initial condition of the errors dynamics to be sufficiently close to zero.

The update law of the delay estimate is characterized by the following "growth condition".

Assumption 5: There exists positive constants $\gamma_{D}>0$ and $M>0$ such that

$$
\begin{gathered}
\dot{\hat{D}}(t)=\gamma_{D} \operatorname{Proj}_{[\underline{D}, \bar{D}]}\left\{\tau_{D}(t)\right\} \\
\text { Case 1 : } \\
\left\{\begin{array}{c}
\left|\tau_{D}(t)\right| \leq M\left(|\tilde{X}(t)|^{2}+\|\tilde{e}(t)\|^{2}+\|\hat{w}(t)\|^{2}+\left\|\hat{w}_{x}(t)\right\|^{2}\right) \\
\text { or Case 2 : } \\
\forall t \geq 0, \quad \tau_{D}(t) \tilde{D}(t) \geq 0 \text { and }\left|\tau_{D}(t)\right| \leq M
\end{array}\right.
\end{gathered}
$$

where, for sake of simplicity and conciseness, |.| represents the usual euclidean norm, $\|$.$\| represents the spatial L^{2}$-norm and $\operatorname{Proj}_{[\underline{D}, \bar{D}]}$ is the standard projector operator on the interval $[\underline{D}, \bar{D}]$.

Theorem 1: Let Assumptions 1-4 hold and consider the closed-loop system consisting of (8)-(10), the control law (17), the update law defined through (18)-(22) and a delay update law satisfying Assumption 5. Let us define

$$
\begin{aligned}
\Gamma(t)= & |\tilde{X}(t)|^{2}+\|e(t)\|^{2}+\|\hat{e}(t)\|^{2}+\left\|\hat{e}_{x}(t)\right\|^{2} \\
& +\tilde{D}(t)^{2}+|\tilde{\theta}(t)|^{2}
\end{aligned}
$$

There exists $\gamma^{*}>0, R>0$ and $\rho>0$ such that, provided the initial state $\left(\tilde{X}(0), e_{0}, \hat{e}_{0}, \hat{e}_{x, 0}, \tilde{\theta}(0), \tilde{D}(0)\right)$ is such that $\Gamma(0)<$ $\rho$ and if $\left(\gamma_{D}, \gamma_{\theta}\right) \in\left(0, \gamma^{*}\right)^{2}$, then

$$
\begin{gathered}
\forall t \geq 0 \quad \Gamma(t) \leq R \Gamma(0) \\
\lim _{t \rightarrow \infty} Y(t)=Y^{r}, \quad \lim _{t \rightarrow \infty} \tilde{X}(t)=0 \quad \text { and } \quad \lim _{t \rightarrow \infty} \tilde{U}(t)=0
\end{gathered}
$$

Comparing again this result with the one established in [5], we observe that the norms of both the estimate $\hat{e}$ and its spatial derivative have replaced the one of the unmeasured true tracking actuator state $e$ in the definition of $\Gamma$ (23). Further, comparing it with the one given in [3], one can notice that the norm of the delay estimation error has been added in (23) and that a significant different consists in the formulation of a very general "growth condition" for the delay update law. This condition is consistant with the one given in in [5] (Case 1). It allows us to update the delay estimate while guaranteeing the stability property (24). The Case 2 in Assumption 5 allows one to consider more sharp update provided that their point is the direction of estimation improvement.

\section{Proof of Theorem 1}

\section{A. Lyapunov Analysis}

In this section, we prove the stability result asserted in Theorem 1 with the use of the following LyapunovKrasovskii functional candidate

$$
\begin{aligned}
V(t) & =\tilde{X}^{T}(t) P(\hat{\theta}) \tilde{X}(t)+b_{1} D \int_{0}^{1}(1+x) \tilde{e}(x, t)^{2} d x \\
& +b_{2} \hat{D}(t)\left[\int_{0}^{1}(1+x) \hat{w}(x, t)^{2} d x+\int_{0}^{1}(1+x) \hat{w}_{x}(x, t)^{2} d x\right] \\
& +b_{2}|\tilde{\theta}(t)|^{2} / \gamma_{\theta}+b_{3} \tilde{D}(t)^{2} / \gamma_{D},
\end{aligned}
$$

where $P$ is defined in assumption (3) and $b_{1}, b_{2}$ and $b_{3}$ are positive constants. This functional is equivalent to $\Gamma$, as will be shown, but reveals helpful in the analysis. Indeed, the factor $(1+x)$ under the integral is handy through integration by parts (and involves directly the expression of the parameter update law (20)). Besides, the variables $(\tilde{X}, \tilde{e}, \hat{w})$ are more suitable for calculations than the original ones $(\tilde{X}, e, \hat{e})$, due to their boundary conditions equal to zero. The equivalence of these two sets of variable could be easily understood considering the transformation (21) along with its inverse

$$
\begin{aligned}
\hat{e}(x, t)= & \hat{w}(x, t)+\hat{D}(t) \int_{0}^{x} K(\hat{\theta}) e^{(A+B K)(\hat{\theta}) \hat{D}(t)(x-y)} B(\hat{\theta}) \\
& \times \hat{w}(y, t) d y+K(\hat{\theta}) e^{(A+B K)(\hat{\theta}) \hat{D}(t) x} \tilde{X}(t)
\end{aligned}
$$

Using these two transformations, the transformed system $(\tilde{X}, \tilde{e}, \hat{w})$ can be written as

$$
\begin{aligned}
& \dot{\tilde{X}}(t)=(A+B K)(\hat{\theta}) \tilde{X}(t)+B(\hat{\theta}) \hat{w}(0, t)+B(\hat{\theta}) \tilde{e}(0, t) \\
&+\tilde{A} X(t)+\tilde{B} u(0, t)-\frac{\partial X^{r}}{\partial \hat{\theta}} \dot{\hat{\theta}}(t) \\
& D \tilde{e}_{t}(x, t)= \tilde{e}_{x}(x, t)-\tilde{D}(t) r(x, t)-D \dot{\hat{D}}(t)(x-1) r(x, t) \\
& \tilde{e}(1, t)= 0 \\
& \hat{D}(t) \hat{w}_{t}(x, t)= \hat{w}_{x}(x, t)-\hat{D}(t) \dot{\hat{D}}(t) p_{0}(x, t)-\hat{D}(t) \dot{\hat{\theta}}(t)^{T} p(x, t) \\
&-\hat{D}(t) \tilde{\theta}(t)^{T} q(x, t)-\hat{D}(t) K(\hat{\theta}) e^{A(\hat{\theta}) \hat{D}(t) x} B(\hat{\theta}) \tilde{e}(0, t) \\
& \hat{w}(1, t)=0
\end{aligned}
$$

where $\tilde{D}(t)=D-\hat{D}(t)$ is the estimation error of the delay, $\tilde{\theta}(t)=\theta-\hat{\theta}(t)$ is the estimation error of the parameter and $\tilde{A}=\sum_{i=1}^{p} A_{i} \tilde{\theta}_{i}(t)$ and $\tilde{B}=\sum_{i=1}^{p} B_{i} \tilde{\theta}_{i}(t)$ are linear quantities in the estimation error of the parameter. 
The quantities $r(x, t)$ and $p_{0}$ and the vectors $q(x, t)$ and $p(x, t)$ can be expressed as functions of $\tilde{X}(t), \tilde{e}(x, t), \hat{w}(x, t)$ and $\hat{w}_{x}(t)$, whose expressions are given in Appendix A ${ }^{2}$. Since our Lyapunov analysis will involve a $H_{1}$ norm of $\hat{w}$, we also need the governing equation of the $\hat{w}_{x}$-system :

$$
\begin{aligned}
& \hat{D} \hat{w}_{x t}(x, t)=\hat{w}_{x x}(x, t)-\hat{D}(t) \dot{\hat{D}}(t) p_{0, x}(x, t) \\
&-\hat{D}(t) \dot{\hat{\theta}}(t)^{T} p_{x}(x, t)-\hat{D}(t) \tilde{\theta}(t)^{T} q_{x}(x, t) \\
&-\hat{D}(t)^{2} K A(\hat{\theta}) e^{A(\hat{\theta}) \hat{D}(t) x} B(\hat{\theta}) \tilde{e}(0, t) \\
& \hat{w}_{x}(1, t)=\hat{D}(t) \dot{\hat{D}}(t) p_{0}(1, t)+\hat{D}(t) \dot{\hat{\theta}}(t) p(1, t) \\
&+\hat{D}(t) \tilde{\theta}(t) q(1, t)+\hat{D}(t) K(\hat{\theta}) e^{A(\hat{\theta}) \hat{D}(t)} B(\hat{\theta}) \tilde{e}(0, t)
\end{aligned}
$$

Taking the time derivative of $V$, with the use of the previous dynamic equation (28)-(32) and of suitable integrations by parts (to use the properties (29) and (30)), we obtain $\dot{V}(t)=-\tilde{X}(t) Q(\hat{\theta}) \tilde{X}(t)+2 \tilde{X}(t)^{T} P B(\hat{\theta})[\hat{w}(0, t)+\tilde{e}(0, t)]$

$$
\begin{aligned}
& +2 \tilde{X}(t)^{T} P(\hat{\theta})[\tilde{A} X(t)+\tilde{B} u(0, t)] \\
& -2 \tilde{X}(t)^{T} P(\hat{\theta}) \frac{\partial X^{r}}{\partial \hat{\theta}} \dot{\hat{\theta}}(t)+\sum_{i=1}^{p} \dot{\hat{\theta}}_{i}(t) \tilde{X}(t)^{T} \frac{\partial P}{\partial \hat{\theta}_{i}} \tilde{X}(t) \\
& +b_{1}\left(-\tilde{e}(0, t)^{2}-\|\tilde{e}(t)\|^{2}-2 \tilde{D} \int_{0}^{1}(1+x) \tilde{e}(x, t) r(x, t) d x\right. \\
& \left.-2 D \dot{\hat{D}}(t) \int_{0}^{1}\left(x^{2}-1\right) \tilde{e}(x, t) r(x, t) d x\right)+b_{2}\left(-\hat{w}(0, t)^{2}\right. \\
& -\|\hat{w}(t)\|^{2}-2 \hat{D}(t) \dot{\hat{D}}(t) \int_{0}^{1}(1+x) \hat{w}(x, t) p_{0}(x, t) d x \\
& -2 \hat{D}(t) \dot{\hat{\theta}}(t)^{T} \int_{0}^{1}(1+x) \hat{w}(x, t) p(x, t) d x \\
& -2 \hat{D}(t) \tilde{\theta}(t)^{T} \int_{0}^{1}(1+x) \hat{w}(x, t) q(x, t) d x \\
& \left.-2 \hat{D}(t) \tilde{e}(0, t) \int_{0}^{1}(1+x) \hat{w}(x, t) K(\hat{\theta}) e^{A(\hat{\theta}) \hat{D} x} B(\hat{\theta}) d x\right) \\
& +b_{2}\left(2 \hat{w}_{x}(1, t)^{2}-\hat{w}_{x}(0, t)^{2}-\left\|\hat{w}_{x}(t)\right\|^{2}\right. \\
& -2 \hat{D}(t) \dot{\hat{D}}(t) \int_{0}^{1}(1+x) \hat{w}_{x}(x, t) p_{0, x}(x, t) d x \\
& -2 \hat{D}(t) \dot{\hat{\theta}}(t)^{T} \int_{0}^{1}(1+x) \hat{w}_{x}(x, t) p_{x}(x, t) d x \\
& -2 \hat{D}(t) \tilde{\theta}(t) \int_{0}^{1}(1+x) \hat{w}_{x}(x, t) q_{x}(x, t) d x \\
& -2 \hat{D}(t)^{2} \tilde{e}(0, t) \int_{0}^{1}(1+x) \hat{w}_{x}(x, t) K A(\hat{\theta}) e^{A(\hat{\theta}) \hat{D} x} B(\hat{\theta}) d x \\
& \left.+\dot{\hat{D}}(t)\left[\int_{0}^{1}(1+x) \hat{w}(x, t)^{2} d x+\int_{0}^{1}(1+x) \hat{w}_{x}(x, t)^{2} d x\right]\right) \\
& -\frac{2 b_{2}}{\gamma_{\theta}} \tilde{\theta}(t)^{T} \dot{\hat{\theta}}(t)-\frac{2 b_{3}}{\gamma_{D}} \tilde{D}(t) \dot{\hat{D}}(t) \\
& \left.-\frac{p}{2}\right)
\end{aligned}
$$

Using the projection operator properties, the update law (18)(19) and denoting $\|f\|_{\infty}=\sup |f(\hat{\theta})|$ with $f: \Pi \rightarrow \mathbb{R}^{m}(m \geq$ 1 ), one can bound this expression as follows

$$
\dot{V}(t) \leq-\left(\underline{\lambda}|\tilde{X}(t)|^{2}+b_{1} \tilde{e}(0, t)^{2}+b_{1}\|\tilde{e}(t)\|^{2}+b_{2} \hat{w}(0, t)^{2}\right.
$$

\footnotetext{
${ }^{2} \mathrm{~A}$ simpler form of these functions could be obtained (e.g. $r(x, t)=$ $\left.\hat{e}_{x}(x, t) / \hat{D}(t)\right)$, but, for Lyapunov analysis purposes, we express them in terms of the variables $\left(\tilde{X}, \tilde{e}, \hat{w}, \hat{w}_{x}\right)$.
}

$$
\begin{aligned}
& \left.+b_{2}\|\hat{w}(t)\|^{2}+b_{2}\left\|\hat{w}_{x}(t)\right\|^{2}\right)+2|\dot{\hat{\theta}}(t)|\left|P(\hat{\theta}) \frac{\partial X^{r}}{\partial \hat{\theta}}\right||\tilde{X}(t)| \\
& +2 b_{2}|h(t)||\tilde{B}||(\tilde{e}(0, t)+\hat{w}(0, t)+K(\hat{\theta}) \tilde{X}(t))| \\
& +2\left|\tilde{X}(t)^{T} P B(\hat{\theta})(\hat{w}(0, t)+\tilde{e}(0, t))\right| \\
& +b_{1}\left(2|\tilde{D}(t)| \int_{0}^{1}(1+x)|\tilde{e}(x, t)||r(x, t)| d x\right. \\
& \left.+2 D|\dot{\hat{D}}(t)| \int_{0}^{1}\left(1-x^{2}\right)|\tilde{e}(x, t)||r(x, t)| d x\right) \\
& +b_{2}\left(2 \hat{D}(t)|\dot{\hat{D}}(t)| \int_{0}^{1}(1+x)|\hat{w}(x, t)|\left|p_{0}(x, t)\right| d x\right. \\
& +2 \hat{D}(t)|\dot{\hat{\theta}}(t)| \int_{0}^{1}(1+x)|\hat{w}(x, t)||p(x, t)| d x+2 \hat{D}(t) \\
& \left.\times|\tilde{e}(0, t)| \int_{0}^{1}(1+x)|\hat{w}(x, t)|\left|K(\hat{\theta}) e^{A(\hat{\theta}) \hat{D}(t) x} B(\hat{\theta})\right| d x\right) \\
& +b_{2}\left(2 \hat{D}(t)|\dot{\hat{D}}(t)| \int_{0}^{1}(1+x)\left|\hat{w}_{x}(x, t)\right|\left|p_{0, x}(x, t)\right| d x\right. \\
& +2 \hat{D}(t)|\dot{\hat{\theta}}(t)| \int_{0}^{1}(1+x)\left|\hat{w}_{x}(x, t)\right|\left|p_{x}(x, t)\right| d x+2 \hat{D}(t)^{2} \\
& \left.\times|\tilde{e}(0, t)| \int_{0}^{1}(1+x)\left|\hat{w}_{x}(x, t)\right|\left|K A(\hat{\theta}) e^{A(\hat{\theta}) \hat{D}(t) x} B(\hat{\theta})\right| d x\right) \\
& +2 b_{2} \hat{w}_{x}(1, t)^{2}+2 b_{2}|\dot{\hat{D}}(t)|\left(\|\hat{w}(t)\|^{2}+\left\|\hat{w}_{x}(t)\right\|^{2}\right) \\
& +\sum_{i=1}^{p}\left|\dot{\hat{\theta}}_{i}(t)\right|\left\|\frac{\partial P}{\partial \hat{\theta}_{i}}\right\|_{\infty}|\tilde{X}(t)|^{2}-\frac{2 b_{3}}{\gamma_{D}} \tilde{D}(t) \dot{\hat{D}}(t)
\end{aligned}
$$

With the inequalities given in Appendix $B$ and the choice of $b_{2} \geq 8\|P B\|_{\infty}^{2} / \underline{\lambda}$, one directly gets, defining $M_{0}=$ $p \max _{1 \leq i \leq p}\left\|\partial P / \partial \hat{\theta}_{i}\right\|_{\infty}$ and $M_{1}=2\left\|\partial X^{r} / \partial \hat{\theta} P\right\|_{\infty}$,

$$
\begin{aligned}
& \dot{V}(t) \leq-\frac{\lambda}{2}|\tilde{X}(t)|^{2}-\left(b_{1}-b_{2}\left(\frac{1}{2}+2 M_{5}\|\theta\|_{\infty}+M_{6}+M_{12}\right.\right. \\
& \left.\left.+4 M_{11}\|\theta\|_{\infty}^{2}+M_{13}\right)\right) \tilde{e}(0, t)^{2}-\frac{b_{2}}{2} \hat{w}(0, t)^{2}-b_{1}\|\tilde{e}(t)\|^{2} \\
& \quad-\left(b_{2}-\frac{b_{2}}{2}\right)\|\hat{w}(t)\|^{2}-\left(b_{2}-\frac{b_{2}}{2}\right)\left\|\hat{w}_{x}(t)\right\|^{2} \\
& -b_{2} \hat{w}_{x}(0, t)^{2}+M_{1}|\dot{\hat{\theta}}(t) \| \tilde{X}(t)| \\
& +b_{1}|\tilde{D}(t)| M_{2}\left(|\tilde{X}(t)|^{2}+\|\tilde{e}(t)\|^{2}+\|\hat{w}(t)\|^{2}+\left\|\hat{w}_{x}(t)\right\|^{2}\right) \\
& +b_{1}|\dot{\hat{D}}(t)| M_{2} \bar{D}\left(|\tilde{X}(t)|^{2}+\|\tilde{e}(t)\|^{2}+\|\hat{w}(t)\|^{2}+\left\|\hat{w}_{x}(t)\right\|^{2}\right) \\
& +b_{2}|\hat{\hat{D}}(t)| M_{3}\left(|\tilde{X}(t)|^{2}+\|\hat{w}(t)\|^{2}+\left\|\hat{w}_{x}(t)\right\|^{2}\right) \\
& +b_{2}|\dot{\hat{\theta}}(t)| M_{4}\left(|\tilde{X}(t)|^{2}+\|\hat{w}(t)\|^{2}+\|\hat{w}(t)\|\right)+b_{2}|\tilde{\theta}(t)| M_{5} \\
& \quad+\left(|\tilde{X}(t)|^{2}+\|\hat{w}(t)\|^{2}+\left\|\hat{w}_{x}(t)\right\|^{2}\right)+b_{2} M_{7}|\dot{\hat{D}}(t)|\left(\hat{w}_{x}(0, t)^{2}\right. \\
& \left.+|\tilde{X}(t)|^{2}+\|\hat{w}(t)\|^{2}+\left\|\hat{w}_{x}(t)\right\|^{2}\right)+b_{2} M_{8}|\dot{\hat{\theta}}(t)|\left(|\tilde{X}(t)|^{2}\right. \\
& \left.+\|\hat{w}(t)\|^{2}+\left\|\hat{w}_{x}(t)\right\|^{2}+\left\|\hat{w}_{x}(t)\right\|\right)+b_{2} M_{9}|\dot{\hat{D}}(t)|^{2}\left(|\tilde{X}(t)|^{2}\right. \\
& \left.+\|\hat{w}(t)\|^{2}\right)+b_{2} M_{10}|\dot{\hat{\theta}}(t)|^{2}\left(|\tilde{X}(t)|^{2}+\left\|\hat{w}_{x}(t)\right\|^{2}+1\right) \\
& +b_{2} M_{11}|\tilde{\theta}(t)|^{2}\left(|\tilde{X}(t)|^{2}+\left\|\hat{w}_{x}(t)\right\|^{2}\right)+M_{0}|\hat{\hat{\theta}}(t) \| \tilde{X}(t)|^{2} \\
& +2 b_{2}|\dot{\hat{D}}(t)|\left(\|\hat{w}(t)\|^{2}+\left\|\hat{w}_{x}(t)\right\|^{2}\right)-\frac{2 b_{3}}{\gamma_{D}} \tilde{D}(t) \dot{\hat{D}}(t) \quad(35)
\end{aligned}
$$


We choose $b_{1} \geq b_{2}\left(\frac{1}{2}+\left(2 M_{5}+4 M_{11}\|\theta\|_{\infty}\right)\|\theta\|_{\infty}+M_{6}\right.$ $\left.+M_{12}+M_{13}\right)$ to make the $\tilde{e}(0, t)^{2}$ term disappear, and define $\eta=\min \left\{\underline{\lambda} / 2, b_{1}, b_{2} / 2\right\}>0$, and

$$
V_{0}(t)=|\tilde{X}(t)|^{2}+\|\tilde{e}(t)\|^{2}+\|\hat{w}(t)\|^{2}+\left\|\hat{w}_{x}(t)\right\|^{2}
$$

From this point, we distinguish the calculations for the two cases of Assumption 5.

1) Case 1 in Assumption 5: In this case, Assumption 5 implies $|\dot{\hat{D}}(t)| \leq \gamma_{D} M V_{0}(t)$. Using this inequality in the previous expression, along with (52), and applying Young's inequality to the arising cubic terms, it is easy to get

$$
\begin{aligned}
\dot{V}(t) & \leq-\left(\eta-|\tilde{D}(t)|\left(b_{1} M_{2}+2 b_{3} M\right)-|\tilde{\theta}(t)| b_{2}\left(2 M_{11}\|\theta\|_{\infty}\right.\right. \\
& \left.\left.+M_{5}\right)-6 \gamma_{\theta} M_{14}\left[M_{0}+M_{1}+b_{2}\left(\gamma_{\theta} M_{10} M_{14}+M_{4}+M_{8}\right)\right]\right) \\
& \times V_{0}(t)+\left(\gamma_{D} M\left(b_{1} M_{2} \bar{D}+b_{2} M_{3}+b_{2} M_{7}+2 b_{2}\right)\right. \\
& \left.+2 \gamma_{\theta} M_{14}\left(M_{0}+M_{1}+b_{2}\left(M_{8}+M_{4}\right)+4 b_{2} \gamma_{\theta} M_{10} M_{14}\right)\right) \\
& \times V_{0}(t)^{2}+b_{2}\left(\gamma_{D}^{2} M^{2} M_{9}+2 \gamma_{\theta}^{2} M_{10} M_{14}^{2}\right) V_{0}(t)^{3} \\
& -b_{2}\left(1-\gamma_{D} M M_{7} V_{0}(t)\right) \hat{w}_{x}(0, t)^{2} \\
& \leq-\left(\eta_{1}-|\tilde{D}(t)| m_{1}-|\tilde{\theta}(t)| m_{2}-\gamma_{\theta} m_{3}\left(\gamma_{\theta}\right)\right) V_{0}(t) \\
& +\left(\gamma_{D} m_{4}+\gamma_{\theta} m_{5}\left(\gamma_{\theta}\right)\right) V_{0}(t)^{2}+m_{6} V_{0}(t)^{3} \\
& -b_{2}\left(1-\gamma_{D} M M_{7} V_{0}(t)\right) \hat{w}_{x}(0, t)^{2}
\end{aligned}
$$

where

$$
\begin{aligned}
m_{1} & =b_{1} M_{2}+2 b_{3} M \\
m_{2} & =b_{2}\left(M_{5}+2 M_{11}\|\theta\|_{\infty}\right) \\
m_{3}\left(\gamma_{\theta}\right) & =6 M_{14}\left(M_{0}+M_{1}+b_{2}\left[\gamma_{\theta} M_{10} M_{14}+M_{4}+M_{8}\right]\right) \\
m_{4} & =M\left(b_{1} M_{2} \bar{D}+b_{2} M_{3}+b_{2} M_{7}+2 b_{2}\right) \\
m_{5}\left(\gamma_{\theta}\right) & =2 M_{14}\left(M_{0}+M_{1}+b_{2}\left(M_{8}+M_{4}\right)+4 b_{2} \gamma_{\theta} M_{10} M_{14}\right) \\
m_{6} & =b_{2}\left(\gamma_{D}^{2} M^{2} M_{9}+2 \gamma_{\theta}^{2} M_{10} M_{14}^{2}\right)
\end{aligned}
$$

Then, we employ the bounds

$$
\begin{aligned}
|\tilde{D}(t)| & \leq \frac{\varepsilon}{2}+\frac{\tilde{D}(t)^{2}}{2 \varepsilon} \\
& \leq \frac{\varepsilon}{2}+\frac{\gamma_{D}}{2 \varepsilon b_{3}}\left(V(t)-\min \left\{\underline{\lambda}, b_{1} \underline{D}, b_{2} \underline{D}\right\} V_{0}(t)\right) \\
& \leq \frac{\varepsilon}{2}+\frac{\gamma_{D}}{2 \varepsilon b_{3}}\left(V(t)-\eta_{1} V_{0}(t)\right) \\
|\tilde{\theta}(t)| & \leq \frac{\varepsilon}{2}+\frac{\gamma_{\theta}}{2 \varepsilon b_{2}}\left(V(t)-\eta_{1} V_{0}(t)\right),
\end{aligned}
$$

which yield

$$
\begin{aligned}
& \dot{V}(t) \leq-\left(\eta-m_{1}\left[\frac{\varepsilon}{2}+\frac{\gamma_{D}}{2 \varepsilon b_{3}} V(t)\right]-m_{2}\left[\frac{\varepsilon}{2}+\frac{\gamma_{\theta}}{2 \varepsilon b_{2}} V(t)\right]\right. \\
& \left.-\gamma_{\theta} m_{3}\left(\gamma_{\theta}\right)\right) V_{0}(t)-\left(\frac{m_{1} \gamma_{D} \eta_{1}}{2 \varepsilon b_{3}}+\frac{m_{2} \gamma_{\theta} \eta_{1}}{2 \varepsilon b_{2}}-\gamma_{D} m_{4}-\gamma_{\theta}\right. \\
& \left.\times m_{5}\left(\gamma_{\theta}\right)-m_{6} V_{0}(t)\right) V_{0}(t)^{2}-b_{2}\left(1-\gamma_{D} M M_{7} V_{0}(t)\right) \hat{w}_{x}(0, t)^{2}
\end{aligned}
$$

Choosing the gain $\gamma_{\theta}$ and the parameter $\varepsilon$ such that

$$
\begin{aligned}
& \gamma_{\theta}<\gamma^{*}=\min \left\{1, \frac{\eta}{m_{3}(1)}\right\} \\
& \varepsilon<\min \left\{\frac{2\left(\eta-\gamma_{\theta} m_{3}\left(\gamma_{\theta}\right)\right)}{m_{1}+m_{2}}, \frac{m_{1} \eta_{1}}{2 b_{3} m_{4}}, \frac{m_{2} \eta_{1}}{2 b_{2} m_{5}\left(\gamma_{\theta}\right)}\right\}
\end{aligned}
$$

and restricting the initial condition so that

$$
\begin{aligned}
& V(0)<\min \left\{\frac{2 \varepsilon}{\frac{\gamma_{D} m_{1}}{b_{3}}+\frac{\gamma_{\theta} m_{2}}{b_{2}}}\left(\eta-\frac{m_{1}+m_{2}}{2} \varepsilon-\gamma_{\theta} m_{3}(1)\right),\right. \\
&\left.\frac{\eta_{1}}{m_{6}}\left(\frac{m_{1} \gamma_{D} \eta_{1}}{2 \varepsilon b_{3}}+\frac{m_{2} \gamma_{\theta} \eta_{1}}{2 \varepsilon b_{2}}-\gamma_{D} m_{4}-\gamma_{\theta} m_{5}\left(\gamma_{\theta}\right)\right), \frac{s \eta_{1}}{\gamma_{D} M M_{7}}\right\}
\end{aligned}
$$

we find that

$$
\dot{V}(t) \leq-\mu_{1}(t) V_{0}(t)-\mu_{2}(t) V_{0}(t)^{2}
$$

where $\mu_{1}$ and $\mu_{2}$ are non-negative functions. Therefore,

$$
\forall t \geq 0, V(t) \leq V(0)
$$

2) Case 2 in Assumption 5: In this case, using the fact that $\dot{\hat{D}}(t) \tilde{D}(t) \geq 0$ in (35), one can obtain, instead of (37),

$$
\begin{aligned}
& \dot{V}(t) \leq-\left(\eta-|\tilde{D}(t)| b_{1} M_{2}-|\tilde{\theta}(t)| b_{2}\left(M_{5}+2 M_{11}\|\theta\|_{\infty}\right)\right. \\
& -\gamma_{D} M\left(b_{1} M_{2} \bar{D}+b_{2} M_{3}+b_{2} M_{7}+2 b_{2}+b_{2} \gamma_{D} M M_{9}\right) \\
& \left.-6 \gamma_{\theta} M_{14}\left(M_{0}+M_{1}+b_{2}\left[4 \gamma_{\theta} M_{10} M_{14}+M_{4}+M_{8}\right]\right)\right) V_{0}(t) \\
& +2 \gamma_{\theta} M_{14}\left(\gamma_{\theta} b_{2} M_{10} M_{14}+M_{0}+M_{1}+b_{2}\left(M_{8}+M_{4}\right)\right) V_{0}(t)^{2} \\
& +2 b_{2} \gamma_{\theta}^{2} M_{10} M_{14}^{2} V_{0}(t)^{3}-b_{2}\left(1-\gamma_{D} M M_{7} V_{0}(t)\right) \hat{w}_{x}(0, t)^{2}
\end{aligned}
$$

Then, the exact same arguments as before can be used. With the bounds (39)-(40), by introducing the quantities $n_{1}, n_{2}, n_{3}\left(\gamma_{D}\right), n_{4}\left(\gamma_{\theta}\right), n_{5}\left(\gamma_{\theta}\right)$ and $n_{6}$, we obtain

$$
\begin{aligned}
& \dot{V}(t) \leq-\left(\eta-n_{1}\left[\frac{\varepsilon}{2}+\frac{\gamma_{D}}{2 \varepsilon b_{3}} V(t)\right]-n_{2}\left[\frac{\varepsilon}{2}+\frac{\gamma_{\theta}}{2 \varepsilon b_{2}} V(t)\right]\right. \\
& \left.-\gamma_{D} n_{3}\left(\gamma_{D}\right)-\gamma_{\theta} n_{4}\left(\gamma_{\theta}\right)\right) V_{0}(t)-\left(\frac{n_{1} \eta_{1} \gamma_{D}}{2 \varepsilon b_{3}}+\frac{n_{2} \eta_{1} \gamma_{\theta}}{2 \varepsilon b_{2}}-\gamma_{\theta}\right. \\
& \left.\times n_{5}\left(\gamma_{\theta}\right)-n_{6} V_{0}(t)\right) V_{0}(t)^{2}-b_{2}\left(1-\gamma_{D} M M_{7} V_{0}(t)\right) \hat{w}_{x}(0, t)^{2}
\end{aligned}
$$

Bounding the gain of both the delay estimate and the parameter estimate (with $\gamma^{*}=\min \left\{1, \frac{\eta}{2 n_{3}(1)}, \frac{\eta}{2 n_{4}(1)}\right\}$ ), taking the parameter $\varepsilon<\min \left\{\frac{2\left(\eta-\gamma_{D} n_{3}\left(\gamma_{D}\right)-\gamma_{\theta} n_{4}\left(\gamma_{\theta}\right)\right)}{n_{1}+n_{2}}, \frac{\eta_{1} n_{2}}{2 b_{2} n_{5} \gamma_{\theta}}\right\}$ and restricting the initial condition of $V$ such that $V(0)<\min \left\{\frac{2 \varepsilon}{\frac{\gamma_{D} n_{1}}{b_{3}}+\frac{\gamma_{\theta} n_{2}}{b_{2}}}\left(\eta-\varepsilon \frac{n_{1}+n_{2}}{2}-\gamma_{D} n_{3}\left(\gamma_{D}\right)-\gamma_{\theta} n_{4}\left(\gamma_{\theta}\right)\right)\right.$, $\left.\frac{\eta_{1}}{n_{6}}\left(\frac{n_{2} \eta_{1} \gamma_{\theta}}{2 \varepsilon b_{2}}-\gamma_{\theta} n_{5}\left(\gamma_{\theta}\right)\right), \frac{\eta_{1}}{\gamma_{D} M M_{7}}\right\}$, we get (41) and (42).

\section{B. Equivalence}

In view of obtaining (24), starting from (42), we prove that the functional $\Gamma$ is equivalent to $V$, i.e. that there exists constants $a>0$ and $b>0$ such that $a V(t) \leq \Gamma(t) \leq b V(t)$. 
Considering (21) and (27), using Young's and CauchySchwartz inequalities, one obtains

$$
\begin{aligned}
\|\hat{e}(t)\|^{2} & \leq r_{1}\|\hat{w}(t)\|^{2}+r_{2}|\tilde{X}(t)|^{2} \\
\left\|\hat{e}_{x}(t)\right\|^{2} & \leq r_{3}\left\|\hat{w}_{x}(t)\right\|^{2}+r_{4}\|\hat{w}(t)\|^{2}+r_{5}|\tilde{X}(t)|^{2} \\
\|\hat{w}(t)\|^{2} & \leq s_{1}\|\hat{e}(t)\|^{2}+s_{2}|\tilde{X}(t)|^{2} \\
\left\|\hat{w}_{x}(t)\right\|^{2} & \leq s_{3}\left\|\hat{e}_{x}(t)\right\|^{2}+s_{4}\|\hat{e}(t)\|^{2}+s_{5}|\tilde{X}(t)|^{2},
\end{aligned}
$$

where $r_{1}, r_{2}, r_{3}, r_{4}, r_{5}, s_{1}, s_{2}, s_{3}, s_{4}$ and $s_{5}$ are sufficiently large positive constants. Then, it is easy to get

$$
\begin{aligned}
\Gamma(t) \leq & \frac{\max \left\{1+3 r_{2}+r_{5}, 3 r_{1}+r_{4}, r_{3}\right\}}{\min \left\{\underline{\lambda}, b_{1} \underline{D}, b_{2} \underline{D}, b_{2} / \gamma_{\theta}, b_{3} / \gamma_{D}\right\}} V(t) \\
V(t) \leq & \max \left\{\bar{\lambda}, 2 b_{1} \bar{D}, 2 b_{2} \bar{D}, b_{2} / \gamma_{\theta}, b_{2} / \gamma_{D}\right\} \\
& \times \max \left\{1+s_{2}+s_{5}, 2+s_{1}+s_{4}, s_{3}\right\} \Gamma(t)
\end{aligned}
$$

which gives the equivalence between the two functionals. This prooves (24) with $R=b / a$.

\section{Convergence Results}

We now conclude using Barbalat's Lemma. Integrating (41) from 0 to $+\infty$, it is easy to get that $|\tilde{X}(t)|$ is square integrable. Further, from (8), one easily obtains

$$
\frac{d|\tilde{X}(t)|^{2}}{d t}=2 \tilde{X}(t)\left(A(\theta) X(t)+B(\theta) u(0, t)-\frac{\partial X^{r}}{\partial \hat{\theta}} \dot{\hat{\theta}}\right)
$$

From (42), it follows that $|\tilde{X}(t)|,\|\tilde{e}(t)\|,\|\hat{w}(t)\|$ and $\left\|\hat{w}_{x}(t)\right\|$ are uniformly bounded. Then, with (43), we obtain the uniform boundedness of $\|\hat{e}(t)\|$. From (17), we conclude that $\tilde{U}(t)$ is uniformly bounded. As $U^{r}(\hat{\theta})$ is continuous from Assumption (2) and $\hat{\theta}(t)$ is uniformly bounded thanks to the projection operators used in the update law, we obtain that $U(t)$ is also bounded for $t \geq 0$. Then, $u(0, t)=U(t-D)$ is uniformly bounded for $t \geq D$. Further, from (52), we get the uniform boundedness of $|\dot{\hat{\theta}}|$. Then, we finally get the uniform boundedness of $d|\tilde{X}(t)|^{2} / d t$ for $t \geq D$. We conclude, by Barbalat's Lemma, that $\tilde{X}(t) \rightarrow 0$ as $t \rightarrow \infty$. Then, it is straightforward that $Y(t) \rightarrow Y^{r}$ as $t \rightarrow \infty$.

Similarly, we obtain

$$
\begin{aligned}
\frac{d \tilde{U}(t)^{2}}{d t}= & 2 \tilde{U}(t)\left(K(\hat{\theta}) e^{A(\hat{\theta}) \hat{D}(t)} \dot{\tilde{X}}(t)+\dot{\hat{D}}(t) G_{0}(t)\right. \\
& \left.+\sum_{i=1}^{p} \dot{\hat{\theta}}_{i}(t) G_{i}(t)+H_{0}(t)\right)
\end{aligned}
$$

where

$$
\begin{aligned}
G_{0}(t)= & K(\hat{\theta})\left[e^{A(\hat{\theta}) \hat{D}(t)} A(\hat{\theta}) \tilde{X}(t)+\int_{0}^{1}(I+A(\hat{\theta}) \hat{D}(1-y))\right. \\
& \times e^{A(\hat{\theta}) \hat{D}(1-y)} B(\hat{\theta}) \hat{e}(y, t) d y \\
& \left.+\int_{0}^{1} e^{A(\hat{\theta}) \hat{D}(t)(1-y)} B(\hat{\theta})(y-1) \hat{e}_{x}(y, t) d y\right] \\
G_{i}(t)= & \frac{\partial K}{\partial \hat{\theta}_{i}}\left[e^{A(\hat{\theta}) \hat{D}(t)} \tilde{X}(t)+\hat{D}(t) \int_{0}^{1} e^{A(\hat{\theta}) \hat{D}(t)(1-y)} B(\hat{\theta})\right. \\
& \times \hat{e}(y, t) d y]+K(\hat{\theta})\left[\hat{D}(t) A_{i} e^{A(\hat{\theta}) \hat{D}(t)} \tilde{X}(t)\right. \\
& +\hat{D}(t) \int_{0}^{1} e^{A(\hat{\theta}) \hat{D}(t)(1-y)}\left(A_{i} \hat{D}(t)(1-y) B(\hat{\theta})+B_{i}\right)
\end{aligned}
$$

$$
\begin{aligned}
& \left.\times \hat{e}(y, t) d y-\hat{D}(t) \int_{0}^{1} e^{A(\hat{\theta}) \hat{D}(t)(1-y)} B(\hat{\theta}) \frac{d u^{r}}{d \hat{\theta}_{i}}(\hat{\theta}) d y\right] \\
H_{0}(t)= & K(\hat{\theta}) \int_{0}^{1} e^{A(\hat{\theta}) \hat{D}(1-y)} B(\hat{\theta}) \hat{e}_{x}(y, t) d y
\end{aligned}
$$

The projection operators employed in the update laws give the uniform boundedness of $\hat{D}(t)$ and $\hat{\theta}$. Then, using the continuity property of the different operators onto $\Pi$ and $[\underline{D}, \bar{D}]$, one can deduce from the previous analysis that $G_{0}(t)$, $G_{1}(t), \ldots, G_{p}(t)$ and $H_{0}(t)$ are uniformly bounded for $t \geq$ $\max \{D, \hat{D}\}$. Then, from (18)-(20) and (5), one obtains the uniform boundedness of $\dot{\hat{D}}(t)$ and $\dot{\hat{\theta}}_{i}(t)$ for $1 \leq i \leq p$. Then, it is easy to conclude that $d \tilde{U}(t)^{2} / d t$ is uniformly bounded. Further, integrating again (41) from 0 to $+\infty,\|\hat{w}(t)\|,|\tilde{X}(t)|$ and consequently, from (43), $\|\hat{e}(t)\|$ are square integrable and so is $\tilde{U}(t)$ as, from (17),

$\tilde{U}(t)=K(\hat{\theta})\left[e^{A(\hat{\theta}) \hat{D}} \tilde{X}(t)+\hat{D}(t) \int_{0}^{1} e^{A(\hat{\theta}) \hat{D}(1-y)} B(\hat{\theta}) \hat{e}(y, t) d y\right]$. Then, one can conclude with Barbalat's Lemma that $\tilde{U}(t) \rightarrow$ 0 as $t \rightarrow \infty$.

\section{Application to the Air/Fuel Ratio control of SPARK-IGNITION ENGINES}

In this section, we present and discuss simulation results obtained using the general control strategy developed above. The treated example, which illustrates the implementation, is the Air/Fuel Ratio (AFR) control in Spark Ignition engines, for which we compare two different delay update laws, satisfying Assumption 5. This application was presented in detail in [3], in an experimental context.

\section{A. Model}

Classically, in SI engines, the AFR is defined as the ratio between the air mass $M_{\text {air }}$ and the fuel mass $M_{\text {fuel }}$ filling the cylinder at each stroke. Here, we use the normalized inverse of the AFR, $\phi=\frac{M_{\text {fuel }}}{M_{\text {air }}} /\left(\frac{M_{\text {fuel }}}{M_{\text {air }}}\right)_{\text {Stoech }}$, that has to be maintained as close as possible to the unity, to maximize the efficiency of exhaust gases after-treatment devices. To accurately achieve this objective, a closed-loop strategy is considered, based on an oxygen sensor located in the exhaust line, whose dynamics can be approximated (see [15]) as

$$
\tau \dot{\phi}(t)=-\phi(t)+\alpha U(t-D)
$$

where $U$ is directly related to the control (the injected mass of fuel). The delay $D$ (between 100 and $600 \mathrm{~ms}$ ) is quite uncertain and mainly accounts for the fact that the sensor is not located directly in the vicinity of the exhaust valve. Finally, the error parameter $\alpha$ (between 0.75 and 1.25) is highly uncertain and represents the errors in the estimation of the in-cylinder air mass and the effects of the injection devices. The proposed strategy can then be applied, with $Y=X=\phi$ and $\theta=\alpha$.

\section{B. Simulation Set-up and Results}

Fig.2 reports the results obtained in simulation for a given set-point (engine speed $N_{e}=1000 \mathrm{rpm}$ and effective mean torque $T=10 \mathrm{Nm}$ ). The value of the corresponding parameters are then $\alpha=1.1$ and $D=0.5 \mathrm{~s}$, initially estimated 


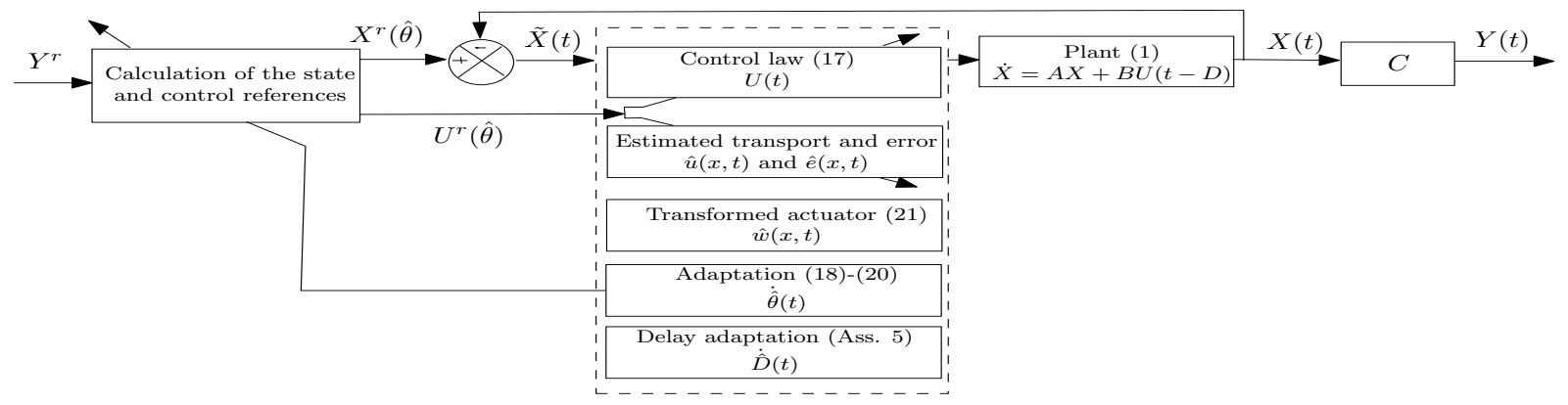

Fig. 1. The proposed adaptive control structure. The closed-loop algorithm uses distributed parameters system (i.e a varying speed waiting-line) which state is used in the adaptive laws (parameter estimate $\hat{\theta}$ update and delay estimate $\hat{D}$ update).

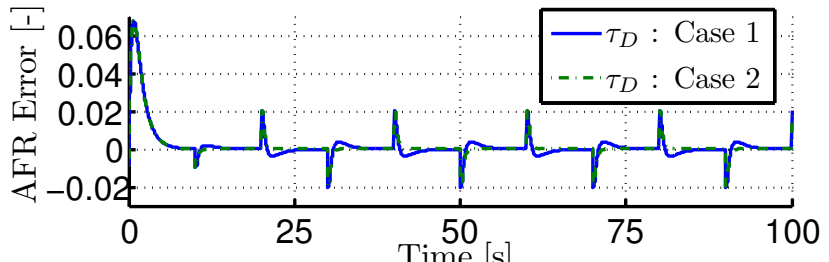

(a) Aime Error

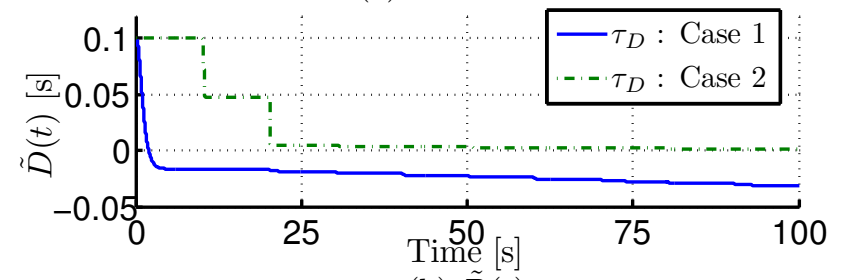

(b) $\tilde{D}(t)$

Fig. 2. Simulation results for a set-point $\left(N_{e}=1000 \mathrm{rpm}\right.$ and $\left.T=10 \mathrm{Nm}\right)$

by $\hat{\theta}(0)=1$ and $\hat{D}(0)=0.4$ s. The tracked trajectory $Y^{r}=X^{r}$ is a periodic input (mean value equal to $1,10 s-$ period and amplitude 0.1 ) which represents the fast switches between rich and lean composition (as usually considered to regenerate the exhaust gases after-treatment devices). The initial state is taken as $X(0)=1$ and we choose the gain $K$ thanks to a $\mathrm{LQR}$ criterion.

We compare two different delay update laws. The first one (referred to as Case 1 in Fig.2) is taken following the certainty equivalence principle and [8] as

$$
\begin{aligned}
\tau_{D}(t)=-\int_{0}^{1}(1+x) \hat{w}(x, t) K(\hat{\theta}) e^{A(\hat{\theta}) \hat{D}(t) x} d x \\
\times(A(\hat{\theta}) \tilde{X}(t)+B(\hat{\theta}) \hat{e}(0, t)),
\end{aligned}
$$

It can be easily shown, using Young's inequality, to satisfy Case 1 in Assumption 5. The second one (referred to as Case 2 in Fig.2) takes advantage of the fact that, for this particular example, the dynamics (18)-(20) and (2) imply the convergence of the parameter $\hat{\alpha}(t)$ to the true value $\alpha$. Then, one can employ a delay update law based on a gradient method (see [11] for a survey of these methods) with respect to the delay estimate. We use here an instantaneous cost function $\hat{D} \longrightarrow|\hat{X}(t, \hat{D})-X(t)|^{2}$, where $\hat{X}(t, \hat{D})$ is the $\hat{D}$ units of time prediction of the system state, starting from $X(t-\hat{D})$ as initial condition and assuming that the delay

value is $\hat{D}$. In this context, this update law satisfies Case 2 in Assumption 5.

The obtained performance of the controller highlight the benefits of our strategy. In both cases, the reference signal is tracked with a good accuracy (see Fig. 2 (a)), and the parameter $\hat{\theta}$ is asymptotically reconstructed. Compared to Case 1, the Case 2 delay update strategy yields an effective asymptotic reconstruction of the true delay of the system. At each step change of the reference signal, a transient occurs during which the delay can be identified.

The presented results have been validated on the simulation software AMESim [6] (presented in [10]). The next step is to validate this control strategy on experimental testbenches. Based on the simulation results presented here and the experiments conducted in [3], one could reasonably expect convergence improvements.

\section{APPENDIX}

\section{A. Appendix A : Expression of $r, p$ and $q$}

$$
\begin{aligned}
& r(x, t)=\frac{\hat{w}_{x}(x, t)}{\hat{D}(t)}+K B(\hat{\theta}) \hat{w}(x, t)+\int_{0}^{x} K(A+B K)(\hat{\theta}) \hat{D}(t) \\
& \times e^{(A+B K)(\hat{\theta}) \hat{D}(t)(x-y)} B(\hat{\theta}) \hat{w}(y, t) d y \\
& +K(A+B K)(\hat{\theta}) e^{(A+B K)(\hat{\theta}) \hat{D}(t) x} \tilde{X}(t) \\
& p_{0}(x, t)=\int_{0}^{x} \hat{w}(y, t)\left[K(\hat{\theta})(I+\hat{D} A(\hat{\theta})(x-y)) e^{A(\hat{\theta}) \hat{D}(x-y)} B(\hat{\theta})\right. \\
& +\hat{D}(t) \int_{y}^{x} K(\hat{\theta})(I+A(\hat{\theta}) \hat{D}(x-\xi)) e^{A(\hat{\theta}) \hat{D}(x-\xi)} B K(\hat{\theta}) \\
& \left.e^{(A+B K)(\hat{\theta}) \hat{D}(\xi-y)} B(\hat{\theta}) d \xi\right] d y+(1-x)\left(\hat{w}_{x}(x, t)\right. \\
& +\hat{D}(t) K B(\hat{\theta}) \hat{w}(x, t)+\hat{D}(t)^{2} \int_{0}^{x} K(A+B K)(\hat{\theta}) \\
& \left.\times e^{(A+B K)(\hat{\theta}) \hat{D}(x-y)} B(\hat{\theta}) \hat{w}(y, t) d y\right)+\hat{D}(t) \int_{0}^{x} K(\hat{\theta}) \\
& \times e^{A(\hat{\theta}) \hat{D}(x-y)} B(\hat{\theta})(1-y) \hat{w}_{x}(y, t) d y+\hat{D}(t)^{2} \int_{0}^{x} \hat{w}(y, t)[K(\hat{\theta}) \\
& \times e^{A(\hat{\theta}) \hat{D}(x-y)} B K B(\hat{\theta})(1-y)+\hat{D}(t) \int_{y}^{x} K(\hat{\theta}) e^{A(\hat{\theta}) \hat{D}(x-\xi)} B(\hat{\theta}) \\
& \left.\times(1-\xi) K(A+B K)(\hat{\theta}) e^{(A+B K)(\hat{\theta}) \hat{D}(\xi-y)} B(\hat{\theta}) d \xi\right] d y
\end{aligned}
$$




$$
\begin{aligned}
& +\left[K A(\hat{\theta}) x e^{A(\hat{\theta}) \hat{D} x}+\int_{0}^{x} K(\hat{\theta})(I+A(\hat{\theta}) \hat{D}(x-y)) e^{A(\hat{\theta}) \hat{D}(x-y)}\right. \\
& \times B K(\hat{\theta}) e^{(A+B K)(\hat{\theta}) \hat{D}_{y}} d y+(1-x) \hat{D} K(A+B K)(\hat{\theta}) \\
& \times e^{(A+B K)(\hat{\theta}) \hat{D} x}+\hat{D}(t)^{2} \int_{0}^{x} K(\hat{\theta}) e^{A(\hat{\theta}) \hat{D}(x-y)} B(\hat{\theta})(1-y) \\
& \left.\times K(A+B K)(\hat{\theta}) e^{(A+B K)(\hat{\theta}) \hat{D}_{y}} d y\right] \tilde{X}(t) \\
& p_{i}(x, t)=\hat{D}(t) \int_{0}^{x} \hat{w}(y, t)\left[\left(\frac{\partial K}{\partial \hat{\theta}_{i}}+K(\hat{\theta}) A_{i} \hat{D}(t)(x-y)\right)\right. \\
& \times e^{A(\hat{\theta}) \hat{D}(t)(x-y)} B(\hat{\theta})+K(\hat{\theta}) e^{A(\hat{\theta}) \hat{D}(t)(x-y)} B_{i} \\
& +\hat{D}(t) \int_{y}^{x}\left[\left(\frac{\partial K}{\partial \hat{\theta}_{i}}+K(\hat{\theta}) A_{i} \hat{D}(t)(x-\xi)\right) e^{A(\hat{\theta}) \hat{D}(t)(x-\xi)} B(\hat{\theta})\right. \\
& \left.\left.+K(\hat{\theta}) e^{A(\hat{\theta}) \hat{D}(t)(x-\xi)} B_{i}\right] K(\hat{\theta}) e^{(A+B K)(\hat{\theta}) \hat{D}(t)(\xi-y)} B(\hat{\theta}) d \xi\right] d y \\
& +\left(\hat { D } ( t ) \int _ { 0 } ^ { x } \left(\left[\frac{\partial K}{\partial \hat{\theta}_{i}}+K(\hat{\theta}) A_{i} \hat{D}(t)(x-y)\right] e^{A(\hat{\theta}) \hat{D}(t)(x-y)} B(\hat{\theta})\right.\right. \\
& \left.+K(\hat{\theta}) e^{A(\hat{\theta}) \hat{D}(t)(x-y)} B_{i}\right) K(\hat{\theta}) e^{(A+B K)(\hat{\theta}) \hat{D}(t) y} d y \\
& \left.+\left[\frac{\partial K}{\partial \hat{\theta}_{i}}+K(\hat{\theta}) A_{i} \hat{D}(t) x\right] e^{A(\hat{\theta}) \hat{D}(t) x}\right) \tilde{X}(t)+\frac{d u^{r}}{d \hat{\theta}_{i}}(\hat{\theta}) \\
& -\hat{D}(t) \int_{0}^{x} K(\hat{\theta}) e^{A(\hat{\theta}) \hat{D}(t)(x-y)} B(\hat{\theta}) \frac{d u^{r}}{d \hat{\theta}_{i}}(\hat{\theta}) d y \\
& -\hat{D}(t) K(\hat{\theta}) e^{A(\hat{\theta}) \hat{D} x} \frac{\partial X^{r}}{\partial \hat{\theta}_{i}} \\
& q_{i}(x, t)=K(\hat{\theta}) e^{A(\hat{\theta}) \hat{D}(t) x}\left(A_{i} X(t)+B_{i} u(0, t)\right)
\end{aligned}
$$

\section{B. Appendix $B$ : Bounds of positive terms involved in the} Lyapunov Analysis

Each variable depending on $\hat{\theta}$, namely, $A, B, P$, etc., is a continuous function of its argument $\hat{\theta}$. Since $\hat{\theta}$ remains in $\Pi$, a closed and bounded subset of $\mathbb{R}^{p}$, by definition of the projector operator $\operatorname{Proj}_{P i}$, each signal admits a finite upper bound. Then, using (48),(49),(50),(51),(32) and the update law (18)-(20), jointly with Young's inequality, CauchySchwartz's inequality and Agmon's inequality $\hat{w}(0, t)^{2} \leq$ $4\left\|\hat{w}_{x}(t)\right\|^{2}$ (with the help of the fact that $\hat{w}(1, t)^{2}=0$ ), one can show that there exist positive constants $M_{1}, M_{2}, \ldots, M_{14}$ independent of initial conditions such that

$$
\begin{aligned}
& 2 \int_{0}^{1}(1+x)|\tilde{e}(x, t) \| r(x, t)| d x \\
& \leq M_{2}\left(|\tilde{X}(t)|^{2}+\|\tilde{e}(t)\|^{2}+\|\hat{w}(t)\|^{2}+\left\|\hat{w}_{x}(t)\right\|^{2}\right) \\
& 2 \int_{0}^{1}\left(1-x^{2}\right)|\tilde{e}(x, t) \| r(x, t)| d x \\
& \leq M_{2}\left(|\tilde{X}(t)|^{2}+\|\tilde{e}(t)\|^{2}+\|\hat{w}(t)\|^{2}+\left\|\hat{w}_{x}(t)\right\|^{2}\right) \\
& 2 \hat{D}(t) \int_{0}^{1}(1+x)\left|\hat{w}(x, t) \| p_{0}(x, t)\right| d x \\
& \leq M_{3}\left(|\tilde{X}(t)|^{2}+\|\hat{w}(t)\|^{2}+\left\|\hat{w}_{x}(t)\right\|^{2}\right) \\
& 2 \hat{D}(t) \int_{0}^{1}(1+x)|\hat{w}(x, t) \| p(x, t)| d x \\
& \leq M_{4}\left(|\tilde{X}(t)|^{2}+\|\hat{w}(t)\|^{2}+\|\hat{w}(t)\|\right) \\
& 2|h(t) \| \tilde{B}||\tilde{e}(0, t)+\hat{w}(0, t)+K(\hat{\theta}) \tilde{X}(t)| \\
& \leq M_{5}|\tilde{\theta}|\left(|\tilde{X}(t)|^{2}+\|\hat{w}(t)\|^{2}+\left\|\hat{w}_{x}(t)\right\|^{2}+\tilde{e}(0, t)^{2}\right)
\end{aligned}
$$

$$
\begin{aligned}
& 2 \hat{D}|\tilde{e}(0, t)| \int_{0}^{1}(1+x)|\hat{w}(x, t)|\left|K(\hat{\theta}) e^{A(\hat{\theta}) \hat{D}(t) x} B(\hat{\theta})\right| d x \\
& \leq M_{6} \tilde{e}(0, t)^{2}+\frac{\|\hat{w}(t)\|^{2}}{2} \\
& 2 \hat{D}(t) \int_{0}^{1}(1+x)\left|\hat{w}_{x}(x, t) \| p_{0, x}(x, t)\right| d x \\
& \leq M_{7}\left(\hat{w}_{x}(0, t)^{2}+|\tilde{X}(t)|^{2}+\|\hat{w}(t)\|^{2}+\left\|\hat{w}_{x}(t)\right\|^{2}\right) \\
& 2 \hat{D}(t) \int_{0}^{1}(1+x)\left|\hat{w}_{x}(x, t) \| p_{x}(x, t)\right| d x \\
& \leq M_{8}\left(|\tilde{X}(t)|^{2}+\|\hat{w}(t)\|^{2}+\left\|\hat{w}_{x}(t)\right\|^{2}+\left\|\hat{w}_{x}(t)\right\|\right) \\
& 2 \hat{w}_{x}(1, t)^{2} \leq M_{9}|\hat{\hat{D}}(t)|^{2}\left(|\tilde{X}(t)|^{2}+\|\hat{w}(t)\|^{2}\right) \\
& +M_{10}|\hat{\hat{\theta}}(t)|^{2}\left(|\tilde{X}(t)|^{2}+\left\|\hat{w}_{x}(t)\right\|^{2}+1\right) \\
& +M_{11}|\tilde{\theta}(t)|^{2}\left(|\tilde{X}(t)|^{2}+\tilde{e}(0, t)^{2}+\left\|\hat{w}_{x}(t)\right\|^{2}\right)+M_{12} \tilde{e}(0, t)^{2} \\
& 2 \hat{D}(t)^{2}|\tilde{e}(0, t)| \int_{0}^{1}(1+x)\left|\hat{w}_{x}(x, t) \| K A(\hat{\theta}) e^{A(\hat{\theta}) \hat{D}(t) x} B(\hat{\theta})\right| d x \\
& \leq M_{13} \tilde{e}(0, t)^{2}+\frac{\left\|\hat{w}_{x}(t)\right\|^{2}}{2} \\
& |\dot{\hat{\theta}}(t)| \leq \gamma_{\theta} M_{14}\left(|\tilde{X}(t)|^{2}+\|\hat{w}(t)\|^{2}+\left\|\hat{w}_{x}(t)\right\|^{2}+|\tilde{X}(t)|\right. \\
& \left.+\|\hat{w}(t)\|+\left\|\hat{w}_{x}(t)\right\|\right)
\end{aligned}
$$

\section{REFERENCES}

[1] Z. Artstein. Linear systems with delayed controls: a reduction. IEEE Transactions on Automatic Control, 27(4):869-879, 1982.

[2] A. T. Bahill. Simple adaptive Smith-predictor for controlling time delay systems. CONTR. SYS. MAG., 3(2):16-22, 1983.

[3] D. Bresch-Pietri, J. Chauvin, and N. Petit. Adaptive backstepping controller for uncertain systems with unknown input time-delay. application to si engines. Proceedings of the 2010 Conference on Decision and Control, 2010.

[4] D. Bresch-Pietri and M. Krstic. Adaptive tracking controller for systems with unknown long delay and unknown parameters in the plant. In American Control Conference, 2009. ACC'09., pages 25752580. IEEE, 2009.

[5] D. Bresch-Pietri and M. Krstic. Adaptive trajectory tracking despite unknown input delay and plant parameters. Automatica, 45(9):20742081, 2009.

[6] LMS IMAGINE. http://www.lmsintl.com.

[7] M. Krstic. Boundary control of PDEs: A course on backstepping designs. Society for Industrial and Applied Mathematics Philadelphia, PA, USA, 2008.

[8] M. Krstic and D. Bresch-Pietri. Delay-adaptive full-state predictor feedback for systems with unknown long actuator delay. In Proceedings of the 2009 conference on American Control Conference, pages 4500-4505, 2009.

[9] M. Krstic and A. Smyshlyaev. Backstepping boundary control for first-order hyperbolic PDEs and application to systems with actuator and sensor delays. Systems \& Control Letters, 57(9):750-758, 2008.

[10] F.A. Lafossas. Application of a new 1d combustion model to gasoline transient engine operation. 2005.

[11] A. O'Dwyer. A survey of techniques for the estimation and compensation of processes with time delay. Report $N^{o} A O D$. 00.03, Dublin Institute of Technology, Irlanda, 2000.

[12] Z. Palmor. Stability properties of Smith dead-time compensator controllers. International Journal of Control, 32:937-49, 1980.

[13] J.-P. Richard. Time-delay systems: an overview of some recent advances and open problems. Automatica, 39(10):1667-1694, 2003.

[14] O. J. M. Smith. A controller to overcome dead time. ISA Journal, 6(2):28-33, 1959.

[15] D. Y. Wang and E. Detwiler. Exhaust oxygen sensor dynamic study. Sensors \& Actuators: B. Chemical, 120(1):200-206, 2006. 